\title{
Face Emotion Recognition is Related to Individual Differences in Psychosis-proneness
}

\section{Citation}

Germine, Laura Thi, and Christine Hooker. 2010. Face emotion recognition is related to individual differences in psychosis-proneness. Psychological Medicine 41(5): 937-947.

\section{Published Version}

doi:10.1017/S0033291710001571

\section{Permanent link}

http://nrs.harvard.edu/urn-3:HUL.InstRepos:5241383

\section{Terms of Use}

This article was downloaded from Harvard University's DASH repository, and is made available under the terms and conditions applicable to Other Posted Material, as set forth at http:// nrs.harvard.edu/urn-3:HUL.InstRepos:dash.current.terms-of-use\#LAA

\section{Share Your Story}

The Harvard community has made this article openly available.

Please share how this access benefits you. Submit a story.

Accessibility 


\title{
Face emotion recognition is related to individual differences in psychosis-proneness
}

\author{
L. T. Germine* and C. I. Hooker \\ Department of Psychology, Harvard University, Cambridge, MA, USA
}

Background. Deficits in face emotion recognition (FER) in schizophrenia are well documented, and have been proposed as a potential intermediate phenotype for schizophrenia liability. However, research on the relationship between psychosis vulnerability and FER has mixed findings and methodological limitations. Moreover, no study has yet characterized the relationship between FER ability and level of psychosis-proneness. If FER ability varies continuously with psychosis-proneness, this suggests a relationship between FER and polygenic risk factors.

Method. We tested two large internet samples to see whether psychometric psychosis-proneness, as measured by the Schizotypal Personality Questionnaire-Brief (SPQ-B), is related to differences in face emotion identification and discrimination or other face processing abilities.

Results. Experiment $1(n=2332)$ showed that psychosis-proneness predicts face emotion identification ability but not face gender identification ability. Experiment $2(n=1514)$ demonstrated that psychosis-proneness also predicts performance on face emotion but not face identity discrimination. The tasks in Experiment 2 used identical stimuli and task parameters, differing only in emotion/identity judgment. Notably, the relationships demonstrated in Experiments 1 and 2 persisted even when individuals with the highest psychosis-proneness levels (the putative highrisk group) were excluded from analysis.

Conclusions. Our data suggest that FER ability is related to individual differences in psychosis-like characteristics in the normal population, and that these differences cannot be accounted for by differences in face processing and/or visual perception. Our results suggest that FER may provide a useful candidate intermediate phenotype.

Received 20 January 2010; Revised 28 June 2010; Accepted 17 July 2010

Key words: Emotion recognition, face processing, individual differences, psychosis-proneness, schizotypy.

\section{Introduction}

Advances in the molecular genetics of schizophrenia increasingly support polygenic risk models based on many genes of small effect (Gottesman \& Shields, 1967; Purcell et al. 2009; Shi et al. 2009; Stefansson et al. 2009). For example, in a recent large-scale genomewide association study, Purcell and colleagues of the International Schizophrenia Consortium (2009) reported that at least one-third of the variance in schizophrenia liability could be explained by a polygenic model involving thousands of commonly occurring alleles. Polygenic models suggest that the genetic liability may manifest as individual differences in specific neural circuits, producing observable neurocognitive intermediate phenotypes (Gottesman \& Gould, 2003; Meyer-Lindenberg \& Weinberger, 2006; Braff et al. 2007; Ivleva et al. 2010).

* Address for correspondence: L. T. Germine, Department of Psychology, Harvard University, 33 Kirkland Street, Cambridge, MA 02138, USA.

(Email: lgermine@fas.harvard.edu)
Based on the criteria proposed by Gottesman \& Gould (2003), deficits in face emotion recognition (FER) provide a potential intermediate phenotype for schizophrenia and related disorders (Gur et al. $2007 a, b)$. FER deficits are consistently related to schizophrenia (Mueser et al. 1997; Mandal et al. 1998; Hooker \& Park, 2002; Kohler \& Brennan, 2004), are observable in early (Edwards et al. 2001) and late psychosis (Mueser et al. 1997), remain after treatment (Herbener et al. 2005), and are related to familial risk (Kee et al. 2004; Bediou et al. 2007). Evidence suggests that FER ability is also highly heritable (Gur et al. $2007 a, b$ ). FER provides the advantage of implicating a well-studied neural network, including the amygdala, superior temporal sulcus and inferior parietal lobe (Adolphs, 2002), whose function can be dissociated from the function of neural networks concerned with static face features (Haxby et al. 2000). Notably, people with schizophrenia spectrum disorders have structural and functional abnormalities in neural regions that support FER processing (Aleman \& Kahn, 2005; Brunet-Gouet \& Decety, 2006), but relatively normal function of neural regions such as 
the fusiform gyrus that support face identity processing (Foxe et al. 2005; Yoon et al. 2006).

Recent evidence suggests that FER deficits are not limited to individuals with schizophrenia, but are more broadly related to psychosis vulnerability (Phillips \& Seidman, 2008). FER deficits have been reported in the first-degree relatives of schizophrenia patients (Kee et al. 2004; Bediou et al. 2007), even where other face processing abilities are unimpaired (Bediou et al. 2007). If FER deficits contribute to the development of psychosis by influencing the development of psychosis-like characteristics, they may also be observable in healthy, high-risk individuals with psychosis-like or subthreshold characteristics (schizotypy or psychosis-proneness). Individuals with high familial risk vary widely in how much they express schizotypal or psychosis-like traits (Kremen et al. 1998; Tsuang et al. 1999; Vollema et al. 2002), so studies of psychometric psychosis-proneness provide a crucial means of addressing the relationship between FER, phenotype and psychosis vulnerability.

Results from studies looking at the relationship between psychometric psychosis-proneness and FER have thus far been mixed or unclear. Some studies have shown FER deficits in individuals high (versus low) in schizotypy or psychosis-proneness (Poreh et al. 1994; Mikhailova et al. 1996; Waldeck \& Miller, 2000; Williams et al. 2007; Aguirre et al. 2008) whereas other studies have not (Toomey \& Schuldberg, 1995; van 't Wout et al. 2004; Jahshan \& Sergi, 2007). However, ceiling effects may have contributed to negative results (e.g. Toomey \& Schuldberg, 1995; Jahshan \& Sergi, 2007) by reducing the ability to detect between-group differences. Sensitive FER tests are needed to detect individual differences in healthy populations.

Furthermore, general cognitive impairment is associated with schizophrenia patients in addition to those at risk; therefore, FER deficits could be part of more generalized deficits in face processing or in visual perception rather than emotion processing (Addington \& Addington, 1998). Of the studies that have used face processing-related control tasks, Poreh et al. (2004) found evidence of general face processing impairment in psychosis-prone individuals, whereas Williams et al. (2007) reported that high psychosisproneness was related to FER impairments but not face identity recognition impairments, based on the Benton Facial Recognition Test (BFRT; although the BFRT may be a suboptimal measure of face discrimination ability; see Duchaine \& Nakayama, 2004). Moreover, differences in procedure or face stimuli between tasks can contribute to misleading or artifactual results. Hence, it is not clear from current research whether the relationship between psychosis-proneness and FER, where observed, is related to more generic processes. Given the possible role of FER as an intermediate phenotype, good behavioral assays in schizophrenia and schizophrenia risk are an important tool, and more research is needed to determine how best to test, characterize and quantify the extent and specificity of ER deficits in individuals with schizophrenia or at risk for schizophrenia.

In addition, as evidence for polygenic models accumulates, it is increasingly important to characterize the relationship between psychosis liability and neurocognition across the continuum. FER differences may, for example, vary linearly with psychosisproneness or only be observable in individuals with the highest levels of psychosis-proneness. Clarifying the nature of this relationship is needed for deciding whether a continuous individual differences model (Claridge, 1997) or a discrete, discontinuous model (e.g. Meehl, 1962, 1990) is most appropriate for characterizing FER as an intermediate phenotype. Thus far, no study has examined the relationship between FER and psychosis liability at intermediate levels of psychosis-proneness.

In two experiments using very large, psychometrically defined samples, we tested the hypothesis that variations across the continuum of psychosis-proneness are related to FER ability but not to other face processing abilities. In Experiment 1, we administered tests of face emotion and face gender identification to extend Bediou et al.'s (2007) finding of selective FER impairments in familial high-risk participants to a sample of participants with varying levels of psychometric risk. In Experiment 2, we replicated our results from Experiment 1 using a test of face emotion and face identity discrimination [the Queen Square Face Discrimination Test (QFDT); Garrido et al. 2009]. These discrimination tasks were designed to be sensitive to individual differences in face processing, closely matched to minimize difficulty or task-related artifacts, and have been shown to rely on specific and dissociable neural subsystems (Pitcher et al. 2008; Garrido et al. 2009).

\section{Experiment 1: Emotion identification versus gender identification}

To determine whether individual differences in face emotion processing performance is related to psychosis-proneness, we administered a face emotion and a face gender identification task to individuals in the normal population with varying levels of psychosis-proneness based on scores from the brief version of the Schizotypal Personality Questionnaire (SPQ-B; Raine \& Benishay, 1995). 
(a)

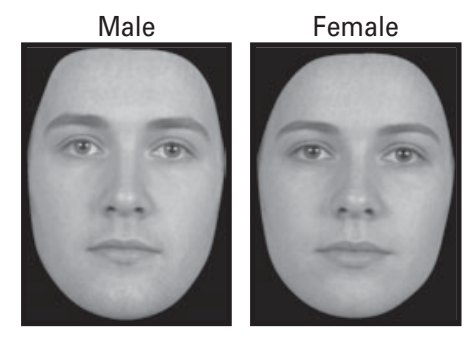

Emotion identification

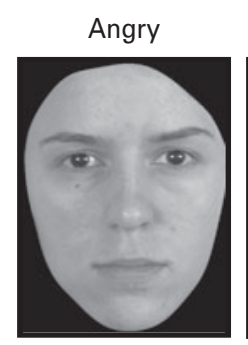

(b)

\section{Emotion discrimination \\ Different}

Identity discrimination

Same

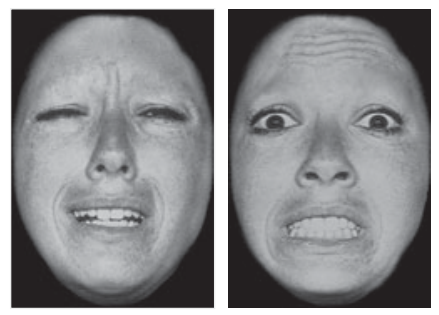

Fig. 1. Stimuli from Experiments 1 and 2. (a) Images from Experiment 1 are shown (Bediou et al. 2007). In the gender identification task, participants had to label each morphed face as male or female. In the emotion identification task, participants had to label each emotion as fearful, angry, disgusted, or happy. Correct responses for each image/trial are shown in italics. (b) Images from Experiment 2 are shown (Garrido et al. 2009). Participants had to judge whether two sequentially presented faces had the same or different emotion (emotion discrimination task) or the same or different identity (identity discrimination task). Correct responses for this image pair in each task are shown in italics.

\section{Method}

\section{Participants}

Subjects were individuals who navigated to the website www.testmybrain.org and clicked on a link labeled 'Recognizing Emotion and Gender from Faces'. Data collected from face processing tests offered on testmybrain.org (different from the ones described here) have been included in a previously published study (Wilmer et al. 2010). There was no specific advertising conducted for the study or the website. Most users arrived at the site through selfgenerated internet searches and by following links posted by other volunteers on social networking websites and blogs. Subjects were given feedback on their performance at the conclusion of the test as incentive for participating. There were no limitations on who could participate in the experiment, but subjects in the reported sample had to meet several criteria. After filling out an online consent form, participants completed a questionnaire assessing demographics, psychiatric, neurological and medical history. Participants were excluded if they endorsed any of the following: age $<16$ or $>65$ years, neurological problems, psychological problems, vision problems, a physical disability that might impact their performance, Asperger's disorder or other autistic spectrum disorder. At the end of the experiment, subjects who indicated that they had had technical problems were also excluded, as were those who may have participated in the experiment before (as indicated by selfreport and/or checking the individual's web browser for a 'cookie' that indicated previous participation).

Our final group comprised 2332 subjects. Table 1 show age, gender and SPQ information for this sample.

\section{Procedure}

All subjects began by completing a test of face gender identification and then a test of face emotion identification, both using morphed face stimuli and adapted from tests previously administered to schizophrenia patients and their relatives (Bediou et al. 2007).

Example stimuli from face emotion and gender identification tests are shown in Fig. 1. In the face gender identification task, faces were created by morphing a gender neutral face with each of four male and four female faces. Each face stimulus contained $20,30,40,50$ or $60 \%$ of the target gender (male or female), yielding 40 face stimuli (eight identities $\times$ five percentage categories). In the face emotion identification task, stimuli were faces morphed between a neutral expression and an emotional expression. There were four different emotional expressions: happy, disgusted, angry, and fearful. Faces were created from 
Table 1. Mean performance and participant information

\begin{tabular}{|c|c|c|c|}
\hline & Range & Mean & S.D. \\
\hline \multicolumn{4}{|l|}{ Experiment $1(n=2332)$} \\
\hline Age (years) & $16-65$ & 29.1 & 11.5 \\
\hline SPQ-B total (all factors) & $0-22$ & $9.2^{\mathrm{a}}$ & 5.1 \\
\hline Interpersonal factor & $0-8$ & 3.8 & 2.6 \\
\hline Cognitive-perceptual factor & $0-8$ & 3.1 & 2.1 \\
\hline Disorganized factor & $0-6$ & 2.3 & 1.9 \\
\hline$\%$ female & 68 & & \\
\hline \multicolumn{4}{|c|}{ Emotion identification (proportion correct) } \\
\hline All emotions ${ }^{\mathrm{b}}$ & $0.17-0.92$ & 0.67 & 0.1 \\
\hline Happiness ${ }^{c}$ & $0.07-1$ & 0.86 & 0.12 \\
\hline Anger ${ }^{c}$ & $0-1$ & 0.61 & 0.17 \\
\hline Disgust $^{c}$ & $0-1$ & 0.53 & 0.19 \\
\hline Fear $^{c}$ & $0-1$ & 0.68 & 0.17 \\
\hline Gender identification $^{\mathrm{d}}$ & $0.43-1$ & 0.81 & 0.08 \\
\hline \multicolumn{4}{|l|}{ Experiment $2(n=1514)$} \\
\hline Age (years) & $16-65$ & 29.3 & 10.6 \\
\hline SPQ-B total (all factors) & $0-22$ & 9.5 & 4.9 \\
\hline Interpersonal factor & $0-8$ & 3.9 & 2.6 \\
\hline Cognitive-perceptual factor & $0-8$ & 3 & 2.1 \\
\hline Disorganized factor & $0-6$ & 2.6 & 1.8 \\
\hline$\%$ female & 62 & & \\
\hline Emotion discrimination $^{\mathrm{d}}$ & $0.4-1$ & 0.81 & 0.08 \\
\hline Identity discrimination $^{\mathrm{d}}$ & $0.23-1$ & 0.77 & 0.09 \\
\hline
\end{tabular}

SPQ-B, Schizotypal Personality Questionnaire - Brief version; S.D., standard deviation.

${ }^{a}$ Mean SPQ-B score from this sample was approximately equal to the mean obtained from a sample of adults with a similar gender distribution (Irwin, 2001: mean $=9.25$, where $63 \%$ were female).

${ }^{\mathrm{b}}$ Proportion correct out of 60 .

${ }^{\mathrm{c}}$ Proportion correct out of 15 .

${ }^{\mathrm{d}}$ Proportion correct out of 40 .

one male and two female face identities. The faces contained $20,30,40,50$ and $60 \%$ of the emotional expression for each identity and each type of facial expression. This yielded 60 face trials (four emotion types $\times$ three identities $\times$ five percentage categories). The original tasks used by Bediou et al. (2007) each contained 10 percentage categories, with trials containing $10-100 \%$ of the target gender or expression. Based on the control data reported by Bediou et al. (2007), the range $20-60 \%$ was chosen for the current experiment to maximize the range of difficulty levels in a minimal number of trials. The different increments of emotion and gender intensities created varying levels of difficulty, and therefore increased the sensitivity of the task to reveal individual differences in performance.

In both tasks, each trial began with a fixation cross for $250 \mathrm{~ms}$, then the face was presented on screen for $1000 \mathrm{~ms}$, followed by the list of answer choices. Participants made a choice between 'male or female' in the face gender test, and 'angry, disgusted, fearful, or happy' in the face emotion test. The answer choices remained on screen for $7 \mathrm{~s}$ or until the participant responded. Participants indicated their response by pressing a key ('m' or 'f'; 'a', 'd', 'f', or ' $h$ '). For each task, participants who failed to respond within the time limit on more than $10 \%$ of trials were excluded from analysis.

After completing both tests, subjects responded to items from the SPQ-B, a measure of psychosis-proneness. The SPQ-B is a 22-item self-report questionnaire that indexes the degree to which an individual has schizophrenia-like cognitive-perceptual (e.g. 'Have you ever noticed a common event or object that seemed to be a special sign for you?'), interpersonal (e.g. 'I feel I have to be on my guard even with my friends'), and disorganized features (e.g. 'I sometimes use words in unusual words').

\section{Results}

A summary of mean performance for this sample is given in Table 1. Paired-sample $t$ test results show that 
participants were more accurate on gender identification as compared with emotion identification $[t(2331)=59.4, p<0.001]$.

Multiple regression was conducted (SPSS version 16.0 ; SPSS Inc., USA) to test the hypothesis that individual differences in psychosis-proneness were related to emotion identification but not to gender identification performance, by using age, participant sex, and SPQ-B scores as predictors of face emotion identification. Previous research has indicated that face processing ability is related to both participant sex and age (McClure, 2000; Bowles et al. 2009), so we controlled for these effects in our analysis. As both SPQ-B scores and age $(r=-0.21)$ and SPQ-B scores and sex $(r=0.06)$ were significantly related in this sample, controlling for age and sex also allowed us to focus on variations in face processing with psychosis-proneness that were not due to variations in age and sex. As expected, the SPQ-B score significantly predicted emotion identification performance $(\beta=-0.09, p<0.001)$, controlling for the effects of $\operatorname{sex}(\beta=-0.18, p<0.001)$ and age $(\beta=-0.07, p<0.01)$. The relationship between psychosis-proneness and emotion identification did not change when gender identification performance was added as a predictor $(\beta=-0.09, p<0.001)$.

Two subgroups were defined by total SPQ-B score such that they roughly represented the bottom and top $10 \%$ of the sample. The top $10 \%$ is traditionally defined as high risk in studies of psychometric schizotypy, and individuals with schizophrenia spectrum disorders such as schizotypal personality disorder are likely to be in the top $10 \%$ of scorers (Raine \& Benishay, 1995) whereas the bottom $10 \%$ is unlikely to contain individual with schizophrenia spectrum diagnoses (Raine, 1991). Individuals with the lowest SPQ-B scores (from 0 to 2, bottom $10 \%$ ) were significantly more accurate than those with the highest SPQ-B scores $(\geqslant 17$, top 9\%) [mean (S.D.) for low SPQ-B scorers: 0.66 (0.1); mean for high SPQ-B scorers $=0.69$ (0.1); independent samples $t$ test: $t(430)=2.7, p<0.01$ ] and corresponded to a Cohen's $d$ effect size of 0.24 . This relationship was not driven entirely by high SPQ-B scorers (those with possible schizophrenia spectrum disorders); SPQ-B scores predicted emotion identification performance even when individuals with high SPQ-B scores (scores of $16 / 22$ or higher) were excluded (2023 participants remaining; $\beta=-0.11, p<0.001)$.

To see whether the observed relationship between psychosis-proneness and face perception was specific to emotion processing, we conducted multiple regression of face gender performance on age, sex and SPQ-B score. The results indicated that although age significantly predicted gender identification performance $(b=0.06, p<0.01)$, SPQ-B score and sex did not (SPQ-B : $b=-0.02, p=0.43$; sex: $b=-0.002, p=0.99$ ).
Accordingly, high and low SPQ-B scorers did not differ significantly in gender identification performance [mean (s.D.) for low SPQ-B scorers $=0.80(0.08)$; mean for high SPQ-B scorers $=0.81(0.08)$; independentsamples $t$ test: $t(430)=1.0, p=0.3$ ].

Scores on the SPQ-B can be divided into three subscales: an interpersonal factor, a cognitive-perceptual factor, and a disorganized factor. These three factors are analogous to the three symptom clusters observed in schizophrenia (Arndt et al. 1991). After controlling for the effects of age and sex, multiple regression analysis revealed that each of the factors predicted emotion performance (interpersonal: $\beta=-0.09$, $p<0.001$; cognitive-perceptual: $\beta=-0.06, p<0.01$; disorganized: $\beta=-0.04, p<0.05)$ but not gender performance (interpersonal: $\beta=-0.03, \quad p=0.23$; cognitive-perceptual; $\beta=0.01, p=0.66$; disorganized: $\beta=-0.02, p=0.27)$.

To identify whether the relationship between SPQ-B score and emotion identification was significantly greater than the relationship between SPQ-B score and gender identification, we used Steiger's $\mathrm{Z1}^{*}$ statistic for comparing two correlation coefficients from the same sample (Steiger, 1980). This analysis showed that the partial correlation between SPQ-B score and emotion identification and SPQ-B score was significantly greater than the partial correlation between SPQ-B score and gender identification $(Z=2.8, p<0.01)$.

Finally, to explore the relationship between SPQ-B scores and identification of specific emotions, we conducted multiple regression with SPQ-B score, age and participant sex as predictors of proportion correct for happy, angry, disgusted and fearful faces separately. Mean performance for individual emotions is shown in Table 1. SPQ-B scores significantly predicted identification of happy faces $(\beta=-0.07, p<0.001)$, angry faces $(\beta=-0.07, p<0.001)$, and fearful faces $(\beta=-0.05, p<0.05)$, but predicted disgusted faces only at the trend level $(\beta=-0.04, p=0.08)$. These results should be interpreted cautiously, however, as we did not have any a priori predictions about the relationship between psychosis-proneness and specific emotions, and the current task was not designed to reveal emotion-specific dissociations.

Fig. 2 shows performance on face emotion and gender identification across the range of SPQ-B scores, illustrating that differences in emotion identification begin to emerge at moderate levels of psychosisproneness.

\section{Experiment 2: Emotion discrimination versus identity discrimination}

There was a significant difference in overall accuracy between the two tasks in Experiment 1, so it is possible 

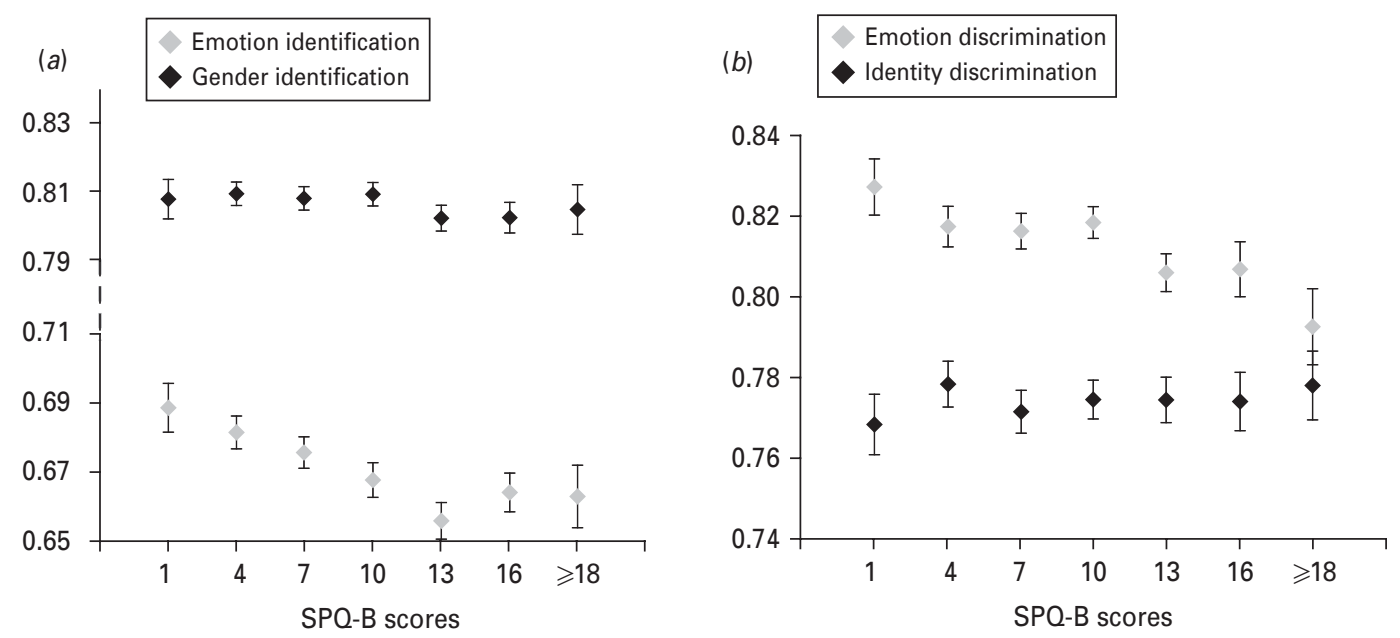

Fig. 2. Task performance and psychosis-proneness. Average proportion correct is shown for individuals at different levels of psychosis-proneness in (a) Experiment 1 and $(b)$ Experiment 2. Although performance on both emotion tasks varied with psychosis-proneness, performance on identity and gender tasks did not. Psychosis-proneness was measured using the brief version of the Schizotypal Personality Questionnaire (SPQ-B; Raine \& Benishay, 1995). For each experiment, proportion correct was binned by SPQ-B score. The median score for each bin is shown, with the exception of the highest bin, which reflects the high end of SPQ-B scorers (scores were positively skewed). Bars reflect \pm 1 standard error. Bins range in size from $n=93$ to $n=495$.

that our findings were the result of differences in task difficulty or differences in task parameters (e.g. there were four response options for the emotion task and only two for the gender task). Differences in difficulty, in particular, pose a significant problem as more difficult tasks are often more sensitive to group differences. Thus, to replicate our findings from Experiment 1 , exclude difficulty-related confounds, and investigate whether or not psychosis-proneness is related to another dimension of face perception (identity processing), we conducted a second experiment using a test of face emotion discrimination and a difficultymatched test of face identity discrimination adapted from the QFDT (Garrido et al. 2009). These tests of identity and emotion discrimination have been used in two prior studies and were shown to tap into dissociable subsystems of face perception, behaviorally and neurally (Pitcher et al. 2008; Garrido et al. 2009). Using a test of emotion discrimination would also allow us to generalize our results from Experiment 1 to face emotion processing more broadly. Whereas emotion discrimination is more purely perceptual, emotion identification relies on other cognitive abilities, such as verbal labeling, that make impairments difficult to interpret (Mandal et al. 1998).

\section{Methods}

\section{Participants}

Subjects were individuals who navigated to the website www.testmybrain.org and clicked on a link labeled 'Recognizing Emotion and Identity from Faces'. Experiments 1 and 2 were never available on our website at the same time, so participant overlap between the two experiments was unlikely to be significant. Exclusion criteria were the same as for Experiment 1, except that we included two additional question prompts to serve as validity checks. Participants were excluded if they responded ' $\mathrm{No}^{\prime}$ to the statement 'I am paying attention to my responses on this questionnaire' or 'Yes' to the statement 'I responded to most of the last 47 questions without reading them'. Our final group comprised 1514 participants. Details of this sample are given in Table 1 . All subjects first completed a test of face identity discrimination followed by a test of face emotion discrimination.

\section{Procedure}

Stimuli were the same for both emotion and identity discrimination tests, and comprised six female models taken from the Ekman \& Friesen (1976) facial affect series expressing either happiness, sadness, surprise, fear, anger or disgust. Pictures were grayscale and cropped, using the same contour to hide the hair and neck. For both tasks, face pairs were presented sequentially for $500 \mathrm{~ms}$ per face with $500 \mathrm{~ms}$ fixation between images. Participants then had up to $7 \mathrm{~s}$ to indicate whether the two faces had the same or different identity (identity discrimination test) or were expressing the same or different emotion (emotion 
discrimination test). Half the trials on each test showed pairs with the same identity/emotion and half the trials showed pairs with different identities/emotions. In the emotion test, identity always varied between the face pairs. In the identity test, emotion always varied between the face pairs. Each test contained 40 trials.

After finishing both tests, subjects again completed items from the SPQ-B, the same measure of psychosisproneness used in Experiment 1.

\section{Results}

Mean performance for this sample is given in Table 1 . Participants were more accurate on emotion discrimination as compared with identity discrimination [paired-samples $t$ test: $t(1513)=14.5, p<0.001]$.

To test the hypothesis that psychosis-proneness was significantly related to emotion discrimination performance, multiple regression was conducted in SPSS (version 16.0 ; 2007) with age, participant sex, and total SPQ-B score as predictors of face emotion discrimination performance. SPQ-B scores in this sample were significantly related to participant age $(r=$ $-0.21)$ but not to sex. Participant sex significantly predicted emotion discrimination performance $(\beta=$ $-0.10, p<0.001)$ whereas age did not $(\beta=-0.014$, $p=0.6$ ). Psychosis-proneness, as measured by the SPQ-B, significantly predicted emotion discrimination performance $(\beta=-0.11, p<0.001)$, even when controlling for identity discrimination performance $(\beta=$ $-0.10, p<0.001)$. Performance was again significantly different between the participants lowest in psychosisproneness (SPQ-B scores $0-2$, bottom $8 \%$ ) and those highest in psychosis-proneness (SPQ-B scores $\geqslant 17$, top 9\%) [mean (S.D.) for low SPQ-B scorers: $0.83(0.8)$; mean for high SPQ-B scorers $=0.79$ (0.1); independent samples $t$ test: $t(261)=3.3, p<0.001$ ], corresponding to a Cohen's $d$ effect size of 0.38. As in Experiment 1, the relationship between SPQ-B score and emotion recognition performance was not being driven entirely by individuals with the highest levels of psychosisproneness and possible schizophrenia spectrum diagnoses. When individuals with scores of $\geqslant 16$ (out of 22) were excluded from analysis, multiple regression again showed that SPQ-B score significantly predicted emotion discrimination (1322 participants remaining; $\beta=-0.07, p<0.05)$.

To see whether differences related to psychosisproneness were limited to emotion discrimination, we conducted multiple regression of face identity discrimination on age, sex, and SPQ-B score. Age and sex predicted identity discrimination performance (age: $\beta=-0.17, p<0.001$; sex: $\beta=-0.14, p<0.001$ ) whereas psychosis-proneness did not $(\beta=-0.03$, $p=0.22)$. This occurred even though overall performance on the identity discrimination task was significantly lower than on the emotion discrimination task, in contrast to Experiment 1 where the emotion task was more difficult. Hence, the observed relationship between psychosis-proneness and emotion processing cannot be explained by difficulty-related confounds.

Multiple regression of emotion discrimination performance on age, sex, and the three factors of the SPQ-B again demonstrated a significant relationship between emotion performance and all three factors (interpersonal: $\beta=-0.07, p<0.05$; cognitive-perceptual: $\beta=-0.10, p<0.001$; disorganized : $\beta=-0.08, p<0.01$ ). Only the interpersonal factor of psychosis-proneness predicted identity discrimination performance (interpersonal: $\beta=-0.05, p<0.05$; cognitive-perceptual: $\beta=0.01, p=0.82$; disorganized : $\beta=-0.02, p=0.54$ ).

In addition, the correlations between SPQ-B score and emotion discrimination and SPQ-B score and identity discrimination were significantly different, based on Steiger's Z1* statistic (1980) for comparing two correlation coefficients from the same sample $(Z=2.3, p<0.01)$.

We did not conduct analyses looking at the relationship between psychosis-proneness and specific emotions for this experiment, as the design (same/ different; six emotion categories) was not conducive to this type of analysis.

Fig. 2 illustrates the relationship between psychosisproneness based on SPQ-B scores and discrimination performance. Consistent with our previous result in Experiment 1, differences in emotion discrimination related to psychosis-proneness are visible at moderate SPQ-B scores.

\section{Discussion}

We have demonstrated in two large samples that increasing psychosis-proneness, as indicated by scores on the SPQ-B (Raine \& Benishay, 1995), is related to reductions in the ability to identify and discriminate facial expressions of emotion. Furthermore, this relationship cannot be accounted for by differences in face processing, visual perception, or a general performance-related factor, as performance on a face gender test (Experiment 1) and a face identity discrimination task (Experiment 2) did not show reductions related to increasing psychosis-proneness. Finally, the relationship between FER and psychosisproneness was significantly predicted by all three factors of our psychosis-proneness measure (interpersonal, cognitive-perceptual, and disorganized). This suggests that FER ability is broadly related to psychosis-like characteristics and not restricted to a 
single dimension of psychosis-proneness, such as positive or negative symptoms.

Our data indicate that the phenotypic expression of subthreshold or psychosis-like features is associated with small, but consistent, differences in the ability to decode facial expressions of emotion in the normal population. These differences are not likely to be clinically significant, but indicate that FER ability varies with individual differences in psychosis-proneness in the normal population. Schizotypal or psychosislike features are related to genetic vulnerability to schizophrenia (Kendler \& Walsh, 1995; Vollema et al. 2002) and elevated schizophrenia risk (Claridge, 1997; Kwapil et al. 1997; Kwapil, 1998; Vollema et al. 2002). Our results suggest that FER deficits observed in schizophrenia and related disorders do not emerge solely as a result of disease-related confounds or secondary characteristics but instead may be a pre-existing or even predisposing neurocognitive feature that varies broadly in the normal population.

We have also shown that FER differences associated with psychosis vulnerability are not associated with more general differences in visual or face processing. Our results are consistent with those of Bediou et al. (2007), who showed that schizophrenia patients and their relatives have FER impairments that are not related to deficits in another type of face processing. This specificity suggests that differences in the neural systems responsible for FER may be related to psychosis vulnerability and the expression of psychosis-like characteristics.

A polygenic model of vulnerability to schizophrenia (Gottesman \& Shields, 1967) suggests that vulnerability-related features may emerge in a continuous fashion across the spectrum of psychosis-proneness (Eysenck, 1960; Chapman \& Chapman, 1980; Raine, 2006). Differences in FER may, for example, reflect the expression of differing numbers of risk-conferring genes and hence were present even at moderate levels of psychosis-proneness in our samples (see Fig. 2). Differences in performance at moderate levels of psychosis-proneness also imply that reductions in FER ability are not attributable solely to early or subthreshold pathology in at-risk participants.

Our study was conducted using a sample recruited entirely on the internet. An increasingly large body of research demonstrates that results from populations tested over the internet are reliable and empirically valid (McGraw et al. 2000; Birnbaum, 2004; Gosling et al. 2004; Kraut et al. 2004; Haworth et al. 2007; Wilmer et al. 2010) and of broad theoretical interest (Owen et al. 2010; Wilmer et al. 2010). A recent analysis of data collected from our website (www.testmybrain. org) on a test of face recognition memory found that performance and reliability from the internet-based sample was the same as from a traditional laboratorybased sample (Wilmer et al. 2010). Our average psychosis-proneness scores were also almost identical to those reported in a community sample with a similar gender distribution (Irwin, 2001). However, despite many precautions taken here to ensure valid data, it was not possible to monitor the performance of each participant in real time, control for biases in selfselection, and verify the accuracy of information provided by participants. These factors most probably added noise to the data and may have interacted with our results in ways that cannot be ascertained based on available data. Ultimately, testing over the internet allowed us to sample a large and diverse population that would not have been practically feasible if this study were conducted in a traditional laboratory setting. This large sample increased our ability to detect small but potentially meaningful effects on both our FER and face processing control tasks.

Variations in face emotion processing have been documented for several psychiatric disorders, including mood disorders (see Leppanen, 2006 for a review) and anxiety disorders (e.g. McClure et al. 2003). Thus, it is possible that our results were partially driven by the overlap between psychosis-like characteristics indexed by the interpersonal factor of the SPQ-B and social anxiety. FER ability was related to multiple subscales of the SPQ-B, however, including scores on the cognitive-perceptual factor, indicating that our results cannot be fully explained by overlap between mood/anxiety symptoms and psychosis-proneness.

Our results recommend an individual differences approach to psychosis-proneness. An individual differences approach has the advantage of complementing the increasing appreciation that schizophrenia and other psychotic disorders are likely to arise from the influence of many common genes of very small effect (Gottesman \& Shields, 1967; Purcell et al. 2009; Shi et al. 2009; Stefansson et al. 2009). The potential relationship between increasing vulnerability to developing psychosis and FER ability suggests that differences in social-emotional processing might contribute to the expression of psychosis-like traits and, ultimately, to psychosis development.

\section{Acknowledgments}

We thank B. Bediou and L. Garrido for providing us with the tasks and stimuli used in Experiments 1 and 2 respectively, and also K. Nakayama for his financial support of www.testmybrain.org, the website used to collect the current dataset. This research was supported by a National Science Foundation (NSF) Graduate Research Fellowship to L. Germine. The NSF had no further role in the research. 


\section{Declaration of Interest}

None.

\section{References}

Addington J, Addington D (1998). Facial affect recognition and information processing in schizophrenia and bipolar disorder. Schizophrenia Research 32, 171-181.

Adolphs R (2002). Neural systems for recognizing emotion. Current Opinion in Neurobiology 12, 169-177.

Aguirre F, Sergi MJ, Levy CA (2008). Emotional intelligence and social functioning in persons with schizotypy. Schizophrenia Research 104, 255-264.

Aleman A, Kahn R (2005). Strange feelings: do amygdala abnormalities dysregulate the emotional brain in schizophrenia? Progress in Neurobiology 77, 283-298.

Arndt S, Alliger R, Andreasen N (1991). The distinction of positive and negative symptoms. The failure of a two-dimensional model. British Journal of Psychiatry 158, 317-322.

Bediou B, Asri F, Brunelin J, Krolak-Salmon P, D'Amato T, Saoud M, Tazi I (2007). Emotion recognition and genetic vulnerability to schizophrenia. British Journal of Psychiatry 191, 126-130.

Birnbaum MH (2004). Human research and data collection via the internet. Annual Review of Psychology 55, 803-832.

Bowles DC, McKone E, Dawel A, Duchaine B, Palermo R, Schmalzl L, Rivolta D, Wilson CE (2009). Diagnosing prosopagnosia: effects of aging, sex, and participant stimulus ethnic match on the Cambridge Face Memory Test and Cambridge Face Perception Test. Cognitive Neuropsychology 26, 423-455.

Braff D, Freedman R, Schork N, Gottesman I (2007). Deconstructing schizophrenia: an overview of the use of endophenotypes in order to understand a complex disorder. Schizophrenia Bulletin 33, 21-32.

Brunet-Gouet E, Decety J (2006). Social brain dysfunctions in schizophrenia : a review of neuroimaging studies. Psychiatry Research: Neuroimaging 148, 75-92.

Chapman L, Chapman J (1980). Scales for rating psychotic and psychotic-like experiences as continua. Schizophrenia Bulletin 6, 477-489.

Claridge G (1997). Theoretical background and issues. In Schizotypy: Implications for Illness and Health (ed. G. Claridge), pp. 3-18. Oxford University Press: New York.

Duchaine BC, Nakayama K (2004). Developmental prosopagnosia and the Benton Facial Recognition Test. Neurology 62, 1219-1220.

Edwards J, Pattison PE, Jackson HJ, Wales RJ (2001). Facial affect and affective prosody recognition in first-episode schizophrenia. Schizophrenia Research 48, 235-253.

Ekman P, Friesen WV (1976). Measuring facial movement. Environmental Psychology and Nonverbal Behavior 1, 56-75.

Eysenck HJ (1960). Classification and the problem of diagnosis. In Handbook of Abnormal Psychology (ed. H. J. Eysenck), pp. 1-31. Pitman: London.

Foxe J, Murray M, Javitt D (2005). Filling-in in schizophrenia: a high-density electrical mapping and source-analysis investigation of illusory contour processing. Cerebral Cortex 15, 1914-1927.

Garrido L, Furl N, Draganski B, Weiskopf N, Stevens J, Tan G, Driver J, Dolan R, Duchaine B (2009). Voxel-based morphometry reveals reduced grey matter volume in the temporal cortex of developmental prosopagnosics. Brain 132, 3443-3455.

Gosling SD, Vazire S, Srivastava S, John OP (2004). Should we trust web-based studies? A comparative analysis of six preconceptions about internet questionnaires. American Psychologist 59, 93-104.

Gottesman I, Gould T (2003). The endophenotype concept in psychiatry: etymology and strategic intentions. American Journal of Psychiatry 160, 636-645.

Gottesman I, Shields J (1967). A polygenic theory of schizophrenia. Proceedings of the National Academy of Sciences USA 58, 199-205.

Gur RE, Calkins ME, Gur RC, Horan WP, et al. (2007a). The consortium on the genetics of schizophrenia: neurocognitive endophenotypes. Schizophrenia Bulletin 33, 49-68.

Gur RE, Nimgaonkar VL, Almasy L, Calkins ME, et al. (2007b). Neurocognitive endophenotypes in a multiplex multigenerational family study of schizophrenia. American Journal of Psychiatry 164, 813-819.

Haworth C, Harlaar N, Kovas Y, Davis O, Oliver B, Hayiou-Thomas M, Frances J, Busfield P, McMillan A, Dale P, Plomin R (2007). Internet cognitive testing of large samples needed in genetic research. Twin Research and Human Genetics 10, 554-563.

Haxby JV, Hoffman EA, Gobbini MI (2000). The distributed human neural system for face perception. Trends in Cognitive Sciences 4, 223-233.

Herbener ES, Hill SK, Marvin RW, Sweeney JA (2005). Effects of antipsychotic treatment on emotion perception deficits in first-episode schizophrenia. American Journal of Psychiatry 162, 1746-1748.

Hooker C, Park S (2002). Emotion processing and its relationship to social functioning in schizophrenia patients. Psychiatry Research 112, 41-50.

Irwin HJ (2001). The relationship between dissociative tendencies and schizotypy: an artifact of childhood trauma? Journal of Clinical Psychology 57, 331-342.

Ivleva E, Morris D, Moates A, Suppes T, Thaker G, Tamminga C (2010). Genetics and intermediate phenotypes of the schizophrenia-bipolar disorder boundary. Neuroscience and Biobehavioral Reviews 34, 897-921.

Jahshan CS, Sergi MJ (2007). Theory of mind, neurocognition, and functional status in schizotypy. Schizophrenia Research 89, 278-286.

Kee K, Horan W, Mintz J, Green M (2004). Do the siblings of schizophrenia patients demonstrate affect perception deficits? Schizophrenia Research 67, 87-94.

Kendler KS, Walsh D (1995). Schizotypal personality disorder in parents and the risk for schizophrenia in siblings. Schizophrenia Bulletin 21, 47-52.

Kohler CG, Brennan AR (2004). Recognition of facial emotions in schizophrenia. Current Opinion in Psychiatry $17,81-86$ 
Kraut R, Olson J, Banaji M, Bruckman A, Cohen J, Couper M (2004). Psychological research online: report of Board of Scientific Affairs' Advisory Group on the Conduct of Research on the Internet. American Psychologist 59, 105-117.

Kremen WS, Faraone SV, Toomey R, Seidman LJ, Tsuang MT (1998). Sex differences in self-reported schizotypal traits in relatives of schizophrenic probands. Schizophrenia Research 34, 27-37.

Kwapil TR (1998). Social anhedonia as a predictor of the development of schizophrenia-spectrum disorders. Journal of Abnormal Psychology 107, 558-565.

Kwapil TR, Miller MB, Zinser MC, Chapman J, Chapman LJ (1997). Magical ideation and social anhedonia as predictors of psychosis proneness: a partial replication. Journal of Abnormal Psychology 106, 491-495.

Leppanen JM (2006). Emotional information processing in mood disorders: a review of behavioral and neuroimaging findings. Current Opinion in Psychiatry 19 34-39.

Mandal MK, Pandey R, Prasad AB (1998). Facial expressions of emotions and schizophrenia: a review. Schizophrenia Bulletin 24, 399-412.

McClure EB (2000). A meta-analytic review of sex differences in facial expression processing and their development in infants, children, and adolescents. Psychological Bulletin 126, 424-453.

McClure EB, Pope K, Hoberman AJ, Pine DS, Leibenluft E (2003). Facial expression recognition in adolescents with mood and anxiety disorders. American Journal of Psychiatry 160, 1172-1174.

McGraw KO, Tew MD, Williams JE (2000). The integrity of Web-delivered experiments: can you trust the data? Psychological Science 11, 502-506.

Meehl PE (1962). Schizotaxia, schizotypy, schizophrenia. American Psychologist 17, 827-838.

Meehl PE (1990). Toward an integrated theory of schizotaxia, schizotypy, and schizophrenia. Journal of Personality Disorders 4, 1-99.

Meyer-Lindenberg A, Weinberger D (2006). Intermediate phenotypes and genetic mechanisms of psychiatric disorders. Nature Reviews. Neuroscience 7, 818-827.

Mikhailova ES, Vladimirova TV, Iznak AF, Tsusulkovskaya EJ (1996). Abnormal recognition of facial expression of emotions in depressed patients with major depression disorder and schizotypal personality disorder. Biological Psychiatry 40, 697-705.

Mueser KT, Penn DL, Blanchard JJ, Bellack AS (1997). Affect recognition in schizophrenia: a synthesis of findings across three studies. Psychiatry: Interpersonal and Biological Processes 60, 301-308.

Owen AM, Hampshire A, Grahn JA, Stenton R, Sajan S, Burns AS, Howard RJ, Ballard CG (2010). Putting brain training to the test. Nature 465, 775-778.

Phillips LK, Seidman LJ (2008). Emotion processing in persons at risk for schizophrenia. Schizophrenia Bulletin 34, 888-903.
Pitcher D, Garrido L, Walsh V, Duchaine B (2008). Transcranial magnetic stimulation disrupts the perception and embodiment of facial expressions. Journal of Neuroscience 28, 8929-8933.

Poreh AM, Whitman RD, Weber M, Ross T (1994). Facial recognition in hypothetically schizotypic college students: the role of generalized poor performance. Journal of Nervous and Mental Disease 182, 503-507.

Purcell S, Wray N, Stone J, Visscher P, O'Donovan M, Sullivan P, Sklar P (2009). Common polygenic variation contributes to risk of schizophrenia and bipolar disorder. Nature 460, 748-752.

Raine A (1991). The SPQ: a scale for the assessment of schizotypal personality based on DSM-III-R criteria. Schizophrenia Bulletin 17, 555-564.

Raine A (2006). Schizotypal personality: neurodevelopmental and psychosocial trajectories. Annual Review of Clinical Psychology 2, 291-326.

Raine A, Benishay D (1995). The SPQ-B: a brief screening instrument for schizotypal personality disorder. Journal of Personality Disorders 9, 346-355.

Shi J, Levinson D, Duan J, Sanders A, Zheng Y, Pe'er I, Dudbridge F, Holmans P, Whittemore A, Mowry B, Olincy A, Amin F, Cloninger C, Silverman J, Buccola N, Byerley W, Black D, Crowe R, Oksenberg J, Mirel D, Kendler K, Freedman R, Gejman P (2009). Common variants on chromosome 6 p22.1 are associated with schizophrenia. Nature 460, 753-757.

Stefansson H, Ophoff R, Steinberg S, Andreassen O, Cichon S, Rujescu D, Werge T, Pietiläinen O, Mors O, Mortensen P, Sigurdsson E, Gustafsson O, Nyegaard M, Tuulio-Henriksson A, Ingason A, Hansen T, Suvisaari J, Lonnqvist J, Paunio T, Børglum A, Hartmann A, Fink-Jensen A, Nordentoft M, Hougaard D, Norgaard-Pedersen B, Böttcher Y, Olesen J, Breuer R, Möller H, Giegling I, Rasmussen H, Timm S, Mattheisen M, Bitter I, Réthelyi J, Magnusdottir B, Sigmundsson T, Olason P, Masson G, Gulcher J, Haraldsson M, Fossdal R, Thorgeirsson T, Thorsteinsdottir U, Ruggeri M, Tosato S, Franke B, Strengman E, Kiemeney L, Melle I, Djurovic S, Abramova L, Kaleda V, Sanjuan J, de Frutos R, Bramon E, Vassos E, Fraser G, Ettinger U, Picchioni M, Walker N, Toulopoulou T, Need A, Ge D, Yoon J, Shianna K, Freimer N, Cantor R, Murray R, Kong A, Golimbet V, Carracedo A, Arango C, Costas J, Jönsson E, Terenius L, Agartz I, Petursson H, Nöthen M, Rietschel M, Matthews P, Muglia P, Peltonen L, St Clair D, Goldstein D, Stefansson K, Collier D (2009). Common variants conferring risk of schizophrenia. Nature 460, 744-747.

Steiger JH (1980). Tests for comparing elements of a correlation matrix. Psychological Bulletin 87, 245-251.

Toomey R, Schuldberg D (1995). Recognition and judgment of facial stimuli in schizotypal subjects. Journal of Communication Disorders 28, 193-203.

Tsuang MT, Stone WS, Faraone SV (1999). Schizophrenia: a review of genetic studies. Harvard Review of Psychiatry 7, 185-207. 
van ‘t Wout M, Aleman A, Kessels RPC, Laroi F, Kahn RS (2004). Emotional processing in a nonclinical psychosis-prone sample. Schizophrenia Research 68, 271-281.

Vollema MG, Sitskoorn MM, Appels MCM, Kahn RS (2002). Does the Schizotypal Personality Questionnaire reflect the biological-genetic vulnerability to schizophrenia? Schizophrenia Research 54, 39-45.

Waldeck TL, Miller LS (2000). Social skills deficits in schizotypal personality disorder. Psychiatry Research $\mathbf{9 3}$, 237-246.
Williams BT, Henry JD, Green MJ (2007). Facial affect recognition and schizotypy. Early Intervention in Psychiatry 1, 177-182.

Wilmer J, Germine L, Chabris C, Chatterjee G, Nakayama K, Williams M, Loken E, Duchaine B (2010) Human face recognition ability is highly heritable. Proceedings of the National Academy of Sciences USA 107, 5238-5241.

Yoon J, D'Esposito M, Carter C (2006). Preserved function of the fusiform face area in schizophrenia as revealed by fMRI. Psychiatry Research 148, 205-216. 